\title{
Formation of a Flat Conductive Polymer Film Using Template-Stripped Gold (TSG) Surface and Surface-Graft Polymerization for Scanning Multiprobe Data Storage
}

\author{
Shinya Yoshida* \\ WPI-Advanced Institute for Materials Research, Tohoku University, \\ 6-6-01 Aramaki-Aza-Aoba, Aoba-ku, Sendai 980-8579, Japan, \\ Takahito Ono \\ Graduate School of Engineering, Tohoku University, \\ 6-6-01 Aramaki-Aza-Aoba, Aoba-ku, Sendai 980-8579, Japan, \\ Masayoshi Esashi \\ WPI-Advanced Institute for Materials Research, Tohoku University, \\ 6-6-01 Aramaki-Aza-Aoba, Aoba-ku, Sendai 980-8579, Japan
}

(Received 6 August 2008; Accepted 22 August 2008; Published 2 September 2008)

\begin{abstract}
A thin conductive polymer recording medium with angstrom-scale surface roughness has been formed on template-stripped gold (TSG) surface by surface-graft polymerization for scanning multiprobe data storage. A gold film is deposited on a mica plate, which is bonded onto the silicon substrate with a gold film using gold-gold direct bonding technique. By removing the mica plate, gold surface with the angstrom-scale surface roughness is formed. A conductive polymer film as a recording medium is deposited on the TSG surface by the surface-graft polymerization. As a result, the polymer film with small roughness is obtained. Thus, the use of the TSG surface is efficient way to form the flat conductive polymer film. Finally, reversible electrical modification on the polymer film is demonstrated using atomic force microscopy. These results show the possibility that this formation method of the conductive polymer film provides rewritable recording media with small roughness for scanning multiprobe high-density data storage. [DOI: 10.1380/ejssnt.2008.202]
\end{abstract}

Keywords: Atomic force microscopy; Nano-scale imaging, measurement, and manipulation technology; Template-stripped metal surface; Conductive polymer; Scanning multiprobe data storage

\section{INTRODUCTION}

Scanning probe data storage systems have been researched for future miniature high-density data storage devices with a recording density beyond $1 \mathrm{~Tb}$ inch $^{-2}[1-3]$. Many research groups have reported various types of recording methods based on scanning tunneling microscopy (STM) $[4,5]$, atomic force microscopy (AFM) [6-10], and scanning near-field optical microscopy (SNOM) [11, 12], etc. Moreover, in order to increase the data transfer rate, parallel data processing using a probe array, i.e. scanning multiprobe data storage systems, has been proposed [13-16]. For scanning multiprobe data storage system, it has been expected that the use of AFM contact mode operation is effective to realize a practical application due to the simplicity of the stage control and recording system integration based on MEMS (MicroElectro Mechanical Systems) technology.

However, this system has representative issues, such as the low wear resistance of the tips and the complexity of the processing circuit. In order to solve above issues, we have developed a recording medium using a conductive polymer for the scanning multiprobe data storage systems $[17-19]$. The recording principle is based on the electrical conductance change of a conductive polymer film, which depends on the redox state of the polymer. The redox state is controlled using an electrochemical reaction. This reaction is caused by applying a voltage between a conductive SPM probe and the conductive polymer film in

*Corresponding author: s-yoshida@mems.mech.tohoku.ac.jp contact operation. Advantages of the conductive polymer recording are as follow. First, it is expected that low stiffness of the conductive polymer can absorb the mechanical energy by its deformation and reduce the wear of the tip. In addition, due to the simple recording method relying on the conductivity change of the conductive polymer, this recording principle does not require complex circuits such as an array of synchronous detection circuits. Thus, the system can be miniaturized and the total volume of the recording system can be reduced due to the signal processing simplicity.

Previously, we have achieved reversible conductance switching and dots pattern definition on a polyaniline (PANI) film as the recording medium [18]. This PANI film was formed by surface-graft polymerization on a gold surface which was deposited by sputtering. Figure 1 shows the schematic figure of the surface-graft polymerization for the PANI film. The PANI chains were grown from the self-assembled monolayer (SAM) of 4-aminothiophenol anchored on the gold surface.

However, the surface of this recording medium is composed from many grains with a large roughness, which is undesirable for the scanning multiprobe data storage system. In this system, all tips should be scanned in contact with the recording medium without precision control of the loading force of the tip. In this contact operation, large surface roughness on the recording medium makes the constant force operation difficult, and increases the wear of both the tips and the medium. In addition, the tip motion cannot follow the rough surface in high speed scan. Thus, the roughness of the medium limits the scan speed, and the error rate in reading and writing data will increase. Therefore, recording media with extremely flat surface is required for the scanning multiprobe data stor- 


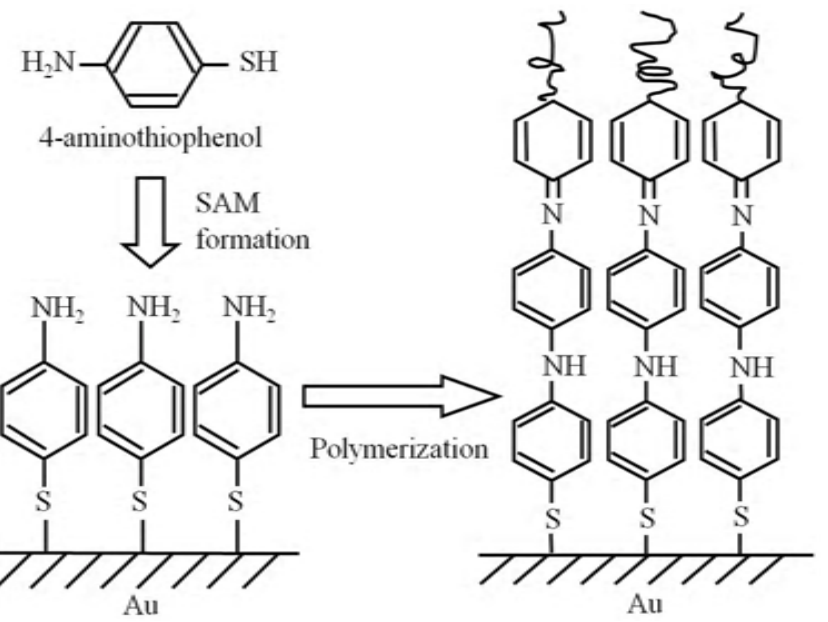

FIG. 1: Scheme of surface-graft polymerization of PANI film. The polymerization of PANI is started from self-assembled monolayer (SAM) fixed on gold surface using gold-thiol group bind.

age system. One of this large surface roughness is due to the roughness of the gold surface as an underlying electrode layer of the film.

In recent years, ultraflat metal surfaces have been fabricated by a template-strip method [20-26]. In particular, template-stripped gold (TSG) surfaces have been of use to form and study highly ordered SAM on large area. The template-strip method utilizes atomically flat surface of a $\mathrm{Si}$ wafer or a cleaved mica as a template to flatten metal surface. This method was generally carried out by following sequence. First, the metal film was deposited on the template with flat surface by sputtering or evaporation. Then, this metal film was transferred from the template onto another substrate. This method can easily produce the metal surfaces with sub-nanometer flatness on a large area. As a base material, rigid substrates such a silicon substrate are preferred because it is easy to form flat surface on a large area without deformation due to the film stress. In addition, the Si substrate is compatible with microfabrication process. Thus, the XYZ stage of the scanning multiprobe data storage system [29] can be fabricated into the Si substrate with the TSG surface. Therefore, the use of the Si substrate has an advantage for the system integration. From these points of view, we choose the Si substrate to transfer the gold film.

In this paper, we form a TSG surface with angstromscale roughness by using the cleaved mica surface as a template, and apply to forming a recording medium for AFM based data storage. As the recording medium, the PANI film is formed on the TSG surface using the surfacegraft polymerization. By comparing between the surface roughness of the PANI films on the TSG and sputtered gold surfaces, it is demonstrated that the TSG can decrease the roughness of the film. Finally, the reversible electrical modification of the flat PANI film on the TSG surface is demonstrated using an AFM system.
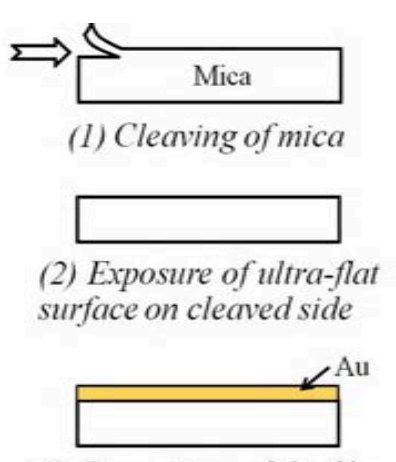

(3) Deposition of Au film by sputtering at $300^{\circ} \mathrm{C}$

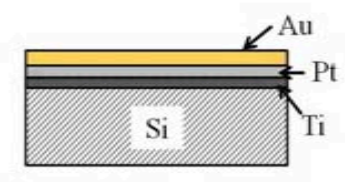

(4) Deposition of Ti, Pt, Au layer by Sputtering

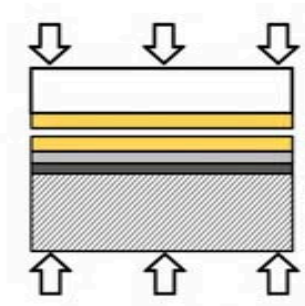

(5) Au-Au direct bonding by application of 35 $\mathrm{MPa}$ at $300^{\circ} \mathrm{C}$

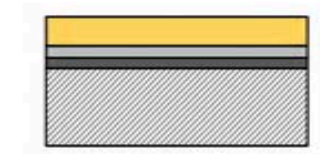

(6) Mechanically strip template to reveal ultraflat gold surface

FIG. 2: Formation procedure of template-stripped gold surface using gold-gold direct bonding. Cleaved mica surface is utilized as a template.

\section{FORMATION OF TEMPLATE STRIPED GOLD SURFACE}

Figure 2 shows the formation procedure of the TSG surface. At the transfer process that the metal film deposited on the template is transferred from the template to a substrate, some organic adhesive layers such as epoxy glue are often used for bonding between the metal film and the substrate. In this study, we employed a gold-gold direct bonding technique [21]. Because any glue is not necessary for the bonding process, the substrates formed by this method have high chemical resistivity. This is advantageous for cleaning the TSG surface with some chemicals and polymerization process of the PANI for a long time.

At first, the silicon substrate was cleaned in piranha solution (1:3 by volume mixture of $30 \%$ hydrogen peroxide and concentrated sulfuric acid) for $30 \mathrm{~min}$. Then, the wafer was rinsed thoroughly with deionized water, dried, and loaded into a vacuum deposition chamber. Then, Ti$\mathrm{Pt}-\mathrm{Au}$ (20-30-300 nm) layers were sputtered on the silicon substrate. On one hand, a mica sheet was cleaved and immediately placed in the vacuum chamber of a sputter deposition equipment. The base pressure of the vacuum chamber was approximately $1.3 \times 10^{-4} \mathrm{~Pa}$. Then, the cleaved mica sheet was heated for $30 \mathrm{~min}$ at $300^{\circ} \mathrm{C}$, and an $\mathrm{Au}$ layer (with a thickness of $1000 \mathrm{~nm}$ ) was deposited on the cleaved side at $300^{\circ} \mathrm{C}$ by sputtering. The mica surface of the template was flat in an atomic scale, therefore, the interface of the $\mathrm{Au}$ film to the mica sheet became very flat. After slow cooling for $5 \mathrm{~h}$, the substrate was taken out from the equipment. Then, the gold-sputtered silicon substrate and the mica sheet were gently placed with these gold films facing each other and touching in hydrostatic press. Then, $35 \mathrm{MPa}$ of pressure was applied to the substrate at $300^{\circ} \mathrm{C}$ for $1 \mathrm{~h}$ in vacuum. With this process, the Au films are bonded together at the Au sur- 

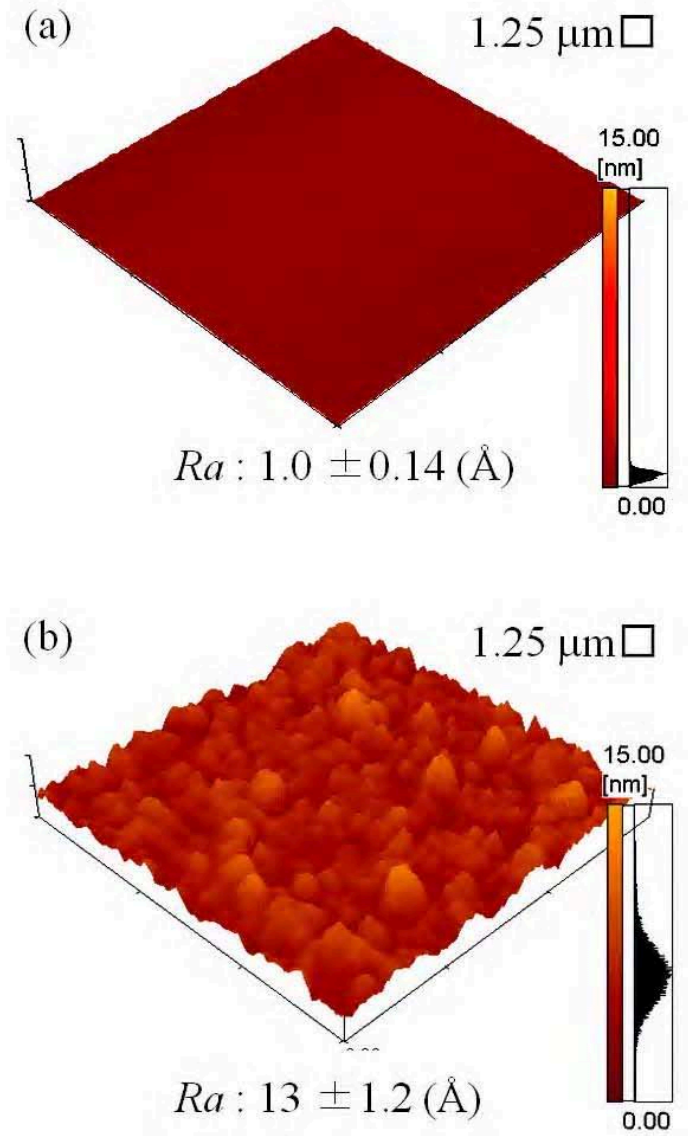

FIG. 3: Topographic images of (a) template-stripped gold surface and (b) sputtered gold surface on $1.25 \mu \mathrm{m} \times 1.25 \mu \mathrm{m}$ square obtained with atomic force microscopy. $R a$ means average and standard deviation of arithmetic mean deviations of the profiles.

faces by metallic bonding. Finally, after slow cooling of the bonded substrate, the mica sheet was mechanically stripped with tweezers. As the result, flat TSG surface in angstrom scale was formed.

The TSG surface was characterized with AFM and STM. AFM characterization was carried out in contact mode in ambient atmosphere using commercial Si cantilever. The spring constant of the cantilever was $0.2 \mathrm{~N} / \mathrm{m}$ and the tip radius was below $10 \mathrm{~nm}$. STM characterization was carried out in ultra high vacuum using a tungsten tip which was acuminated by electropolishing. Figures 3 (a) and (b) show topographic images of TSG and a sputtered gold surface on $1.25 \mu \mathrm{m} \times 1.25 \mu \mathrm{m}$ squares obtained with AFM. The sputtered gold surface shows large surface roughness due to gold grains. In contrast, the TSG surface image shows smaller surface roughness than that of the sputtered gold surface. $R a$ (average and standard deviation of arithmetic mean deviations of the profiles) of the TSG surface and the sputtered gold surface are $1.0 \pm 0.14 \AA$ and $13 \pm 1.2 \AA$, respectively.

Figure 4 (a) shows the topographic image of the TSG surface on an area of $200 \mathrm{~nm} \times 200 \mathrm{~nm}$ squares taken by STM. $R a$ in this area is $0.55 \pm 0.09 \AA$. This $R a$ value is smaller than that in the AFM measurement on $1.25 \mu \mathrm{m} \times 1.25 \mu \mathrm{m}$ squares, because the surface roughness with a long period is not taken into account on the mea-

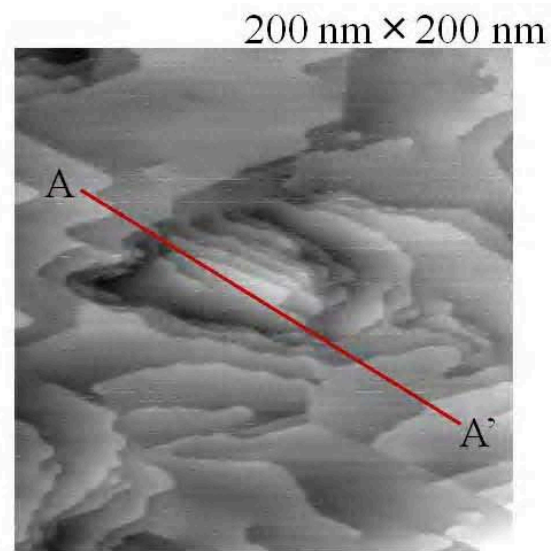

(a)

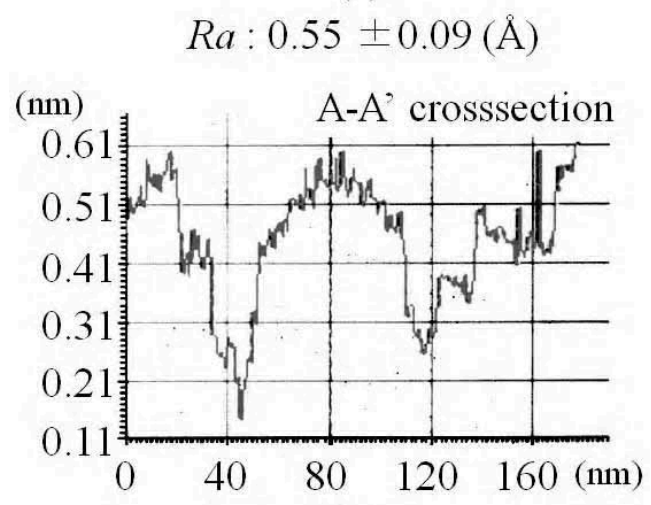

(b)

FIG. 4: (a) Topographic image of template-stripped gold surface on $200 \mathrm{~nm} \times 200 \mathrm{~nm}$ taken by scanning tunneling microscopy. (b) the profile along the line A-A' in Fig. 3(a).

surement of the small scanning area $(200 \mathrm{~nm} \times 200 \mathrm{~nm}$ squares). Crystalline terraces and steps with 1-2 $\AA$ height are observed. The period of stacked layer of mica is known to be 7-10 $\AA$. Thus, these crystalline terrace and step were formed not by pattern transfer of the shape of the mica surface, but by crystallization of the Au due to heating at $300^{\circ} \mathrm{C}$ in the sputtering or the bonding process. Figure 4 (b) shows the profile along the A-A' line in Fig. 4 (a). This shows maximum height of the profile is approximately $4.5 \AA$. From these results, it is indicated that atomically flat gold surface can be obtained by the template-stripped method using the gold-gold direct bonding.

\section{FORMATION OF A FLAT CONDUCTIVE POLYMER FILM ON TSG SURFACE USING SURFACE-GRAFT POLYMERIZATION}

PANI was chosen as the conductive polymer, of which conductivity depends on the redox level, as shown in Fig. 5 [27]. A PANI film was formed on the TSG surface by the surface-graft polymerization, as shown in Fig. 1.

First, the TSG substrate was immersed into an ethanol solution of $1 \mathrm{mM} 4$-aminothiophenol for $30 \mathrm{~h}$ at room temperature, and then rinsed with ethanol and dried. As the result, the SAM of the 4-aminothiophenol was built on the TSG surface, which became a binding material 


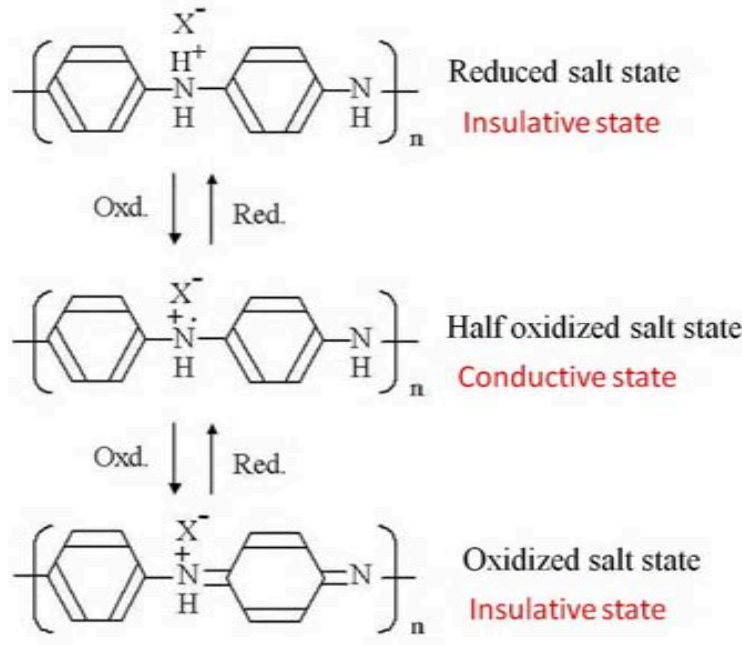

FIG. 5: Molecular structures involved in conductive states of PANI. Conductivity of PANI depends on the redox level. $\left(\mathrm{X}^{-}\right.$: anion $)$

between molecular chains of the PANI and the TSG surface. Then, the polymerization of the PANI was carried out to the SAM by oxidative polymerization of aniline. The PANI molecular chains were bound and grown on the TSG surface by immersing the substrate in $1 \mathrm{M} \mathrm{HCl}$ solution containing $0.15 \mathrm{M}$ aniline and $0.1 \mathrm{M}\left(\mathrm{NH}_{4}\right)_{2} \mathrm{~S}_{2} \mathrm{O}_{8}$ at $0^{\circ} \mathrm{C}$ for $20 \mathrm{~h}$. As a result, the PANI film was formed on the ultra-flat TSG surface. This PANI film consisted of physically absorbed PANI chains and chemically bonded chains to the gold surface through thiol group. Then, the substrate was immersed in a concentrated ammonia water for $1 \mathrm{~h}$ in order to remove dopant from the PANI film. After that, the physically absorbed PANI was removed in N-methyl-2-pyrrolidinone using an ultrasonic bath and rinsed in deionised water. Finally, the PANI film was doped by immersing in a $1 \mathrm{M} \mathrm{HCl}$ solution, and rinsed in deionised water and dried. This sequence of the PANI polymerization was repeated three times in order to obtain the PANI film with the desired thickness. As the result, the PANI film with a thickness of approximately $20 \mathrm{~nm}$ was obtained. The film had strong adhesion to the substrate due to the strong chemical bonds of the thiol group to the gold surface.

The PANI film on the TSG surface was characterized with AFM. Figures 6 (a) and (b) are the topographic images of the PANI films on the TSG surface and sputtered gold surface, respectively. $R a$ of the PANI films on the TSG surface and the sputtered gold surface are $3.5 \pm 0.37 \AA$ and $14 \pm 1.7 \AA$, respectively. As compared to roughness of the TSG surface as shown in Fig. 3, the formed PANI surface on the TSG became slightly rougher. After the formation of the PANI film, the surface roughness $(R a)$ increased from $1.0 \AA$ to $3.5 \AA$. However, the roughness of the PANI film on the TSG surface was smaller than that on the sputtered gold surface. $R a$ of the PANI film on the TSG surface is approximately fourth as compared to that on the sputtered gold surface. Thus, the use of the TSG method is effective way to improve the surface roughness of polymerized surface on it.

In order to investigate what causes the roughness of
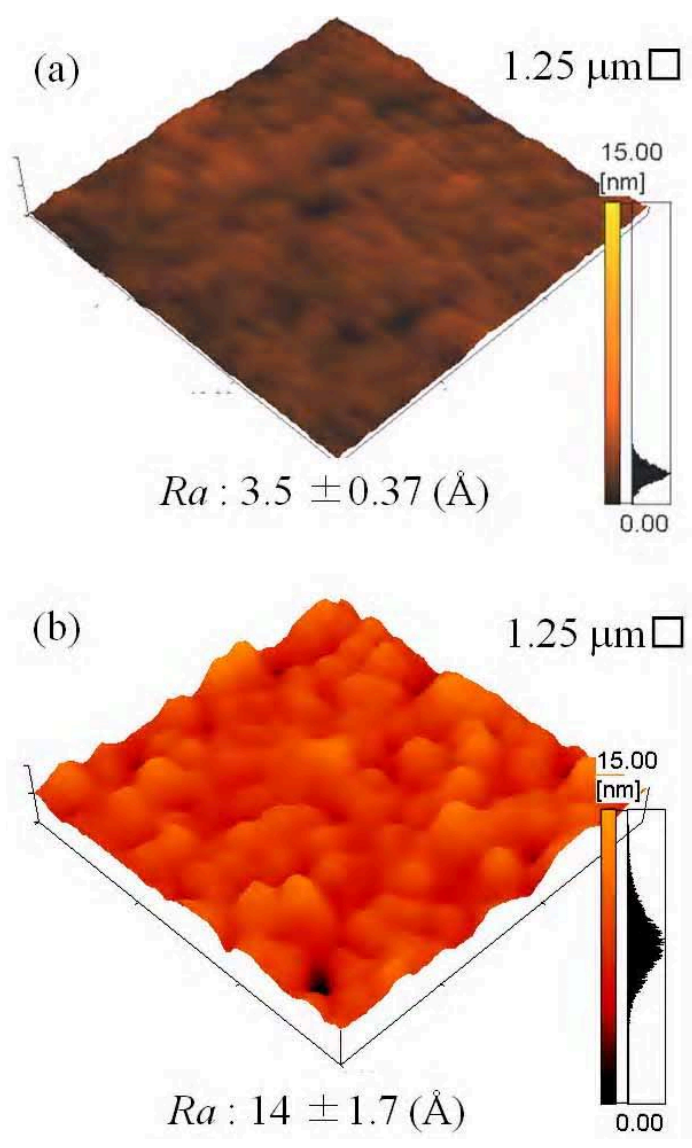

FIG. 6: Topographic images of the PANI films (a) on the TSG surface and (b) on sputtered gold surface, respectively. $R a$ means average and standard deviation of arithmetic mean deviations of the profiles.

the conductive polymer film, the surface morphology was measured. Figure 7 shows the topographic image of the PANI film on the TSG surface in $400 \mathrm{~nm} \times 400 \mathrm{~nm}$ square. It can be seen that the PANI film consists of many grains with sizes of 50-100 nm. The PANI molecular chain has intramolecular polarity between the dopant and the nitrogen atom, as shown in Fig. 5. This polarity raises strong attractive interaction among the PANI molecular chains. In addition, the PANI chains have many aromatic rings. Thus, $\pi-\pi$ interaction of aromatic rings also tends to be induced among the chains. Therefore, it is considered that these PANI grains are caused by aggregation of the PANI.

\section{ELECTRICAL MODIFICATION EXPERIMENTAL}

The electrical modification of the flat PANI film was demonstrated using the AFM system in atmosphere. Figure 8 shows the schematic illustration of the experimental setup. The sample was put on a PZT scanner. A Ptcoated cantilever was scanned in contact with the PANI film surface. In the modification experiment, a DC voltage was applied to the sample against the grounded probe while the probe was scanned on the sample. The sequence of electrical modification is shown in Fig. 9. First, the 


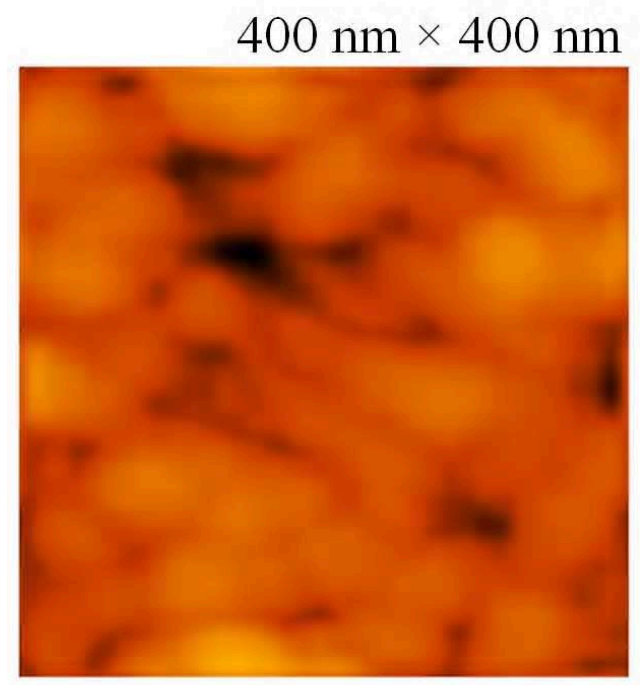

FIG. 7: Topographic image of the PANI film formed by surface-graft polymerization on the TSG surface.

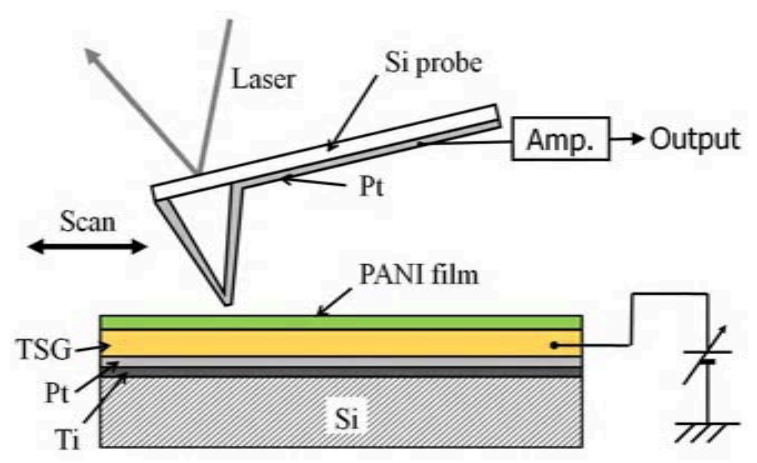

FIG. 8: Schematic illustration of electrical modification experimental setup for the PANI film on the TSG surface.

PANI film was electrically modified by applying a positive voltage of $2 \mathrm{~V}$ on the rectangle area. Then, a negative voltage of $-2 \mathrm{~V}$ was applied to the area smaller than previously modified area. The process was repeated again on another smaller inner rectangle area with $2 \mathrm{~V}$ applied. In order to measure the conductivity change of the conductive polymer, conductance images were taken by applying a voltage of $0.02 \mathrm{~V}$ after the electrical modification. The flowing current was measured by an amplifier, and the conductance image was obtained by mapping the current.

Figure 10 shows the conductance image taken from the current variation under the application of $0.02 \mathrm{~V}$ during the scanning after the sequential modification. In this image, the dark part shows the low-conductance areas. It is shown that applying positive voltages decreased the conductivity of PANI, and negative voltages increased. Thus, it is indicated from the electrical modification result that the conductivity of PANI film can be reversibly changed, depending on the polarity of the applied voltage to PANI films using conductive AFM tip.

Figures 11(a) and (b) show the simultaneously-obtained conductance and topographic images of the surface of the

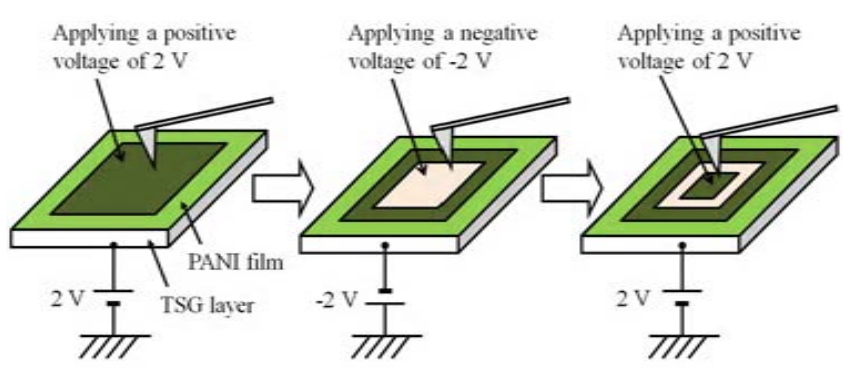

FIG. 9: Electrical modification sequence of PANI film. After the electrical modification, conductance images were taken by applying a voltage of $0.02 \mathrm{~V}$ for investigation of the conductivity change of the PANI film.

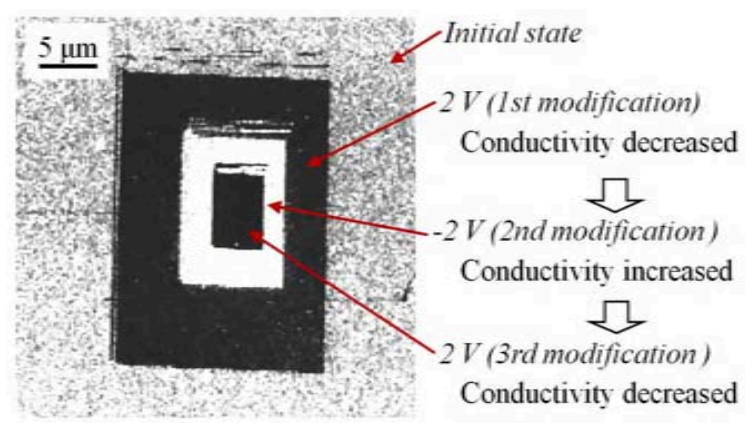

FIG. 10: Conductance image acquired from the current variation under the application of $0.02 \mathrm{~V}$ during the scanning on the PANI film after the sequential modification. (The dark part shows the low-conductance areas.)

PANI film after the electrical modification, respectively. In contrasted to the conductance image, no change is observed in the topographic image of the film. This fact means that the reversible conductance change of the PANI film is not caused by change of the film thickness, but caused by the change of the electrical conductance of the film. The conductance change of the recording medium without the topographic change is considered to be advantageous for high-speed scan of the recording tips.

Figure 12 shows the current profile of the conductance image at line A-A' after the electrical modification. In the current profile figure, B and B' lines correspond to current averages of the $2 \mathrm{~V}$ and $-2 \mathrm{~V}$ modified area, respectively. It can be seen that the conductivity on the modified area by negative voltage is approximately 20 times larger than that by positive voltage. If supposed that the contact area between the AFM tip and the surface of the PANI film is $400 \pi \mathrm{nm}^{2}$, and also the influence of the contact resistance is not taken into account, the average conductivity of the modified area with a modification voltage of $2 \mathrm{~V}$ is calculated to be around $1-2 \times 10^{-5} \mathrm{~S} \mathrm{~cm}^{-1}$. On the other hand, the average conductivity with a modification voltage of $-2 \mathrm{~V}$ is approximately $2-4 \times 10^{-4} \mathrm{~S} \mathrm{~cm}^{-1}$. It is reported that the conductivity of the PANI is ranging from 15 to $10^{-5} \mathrm{~S} \mathrm{~cm}^{-1}$, depending on the redox level of the PANI [28]. Thus, it is considered reasonable that the PANI modified with a voltage of $2 \mathrm{~V}$ became insulative state (oxidized salt state). In contrast, the conductivity with a modification voltage of $-2 \mathrm{~V}$ is lower than the 


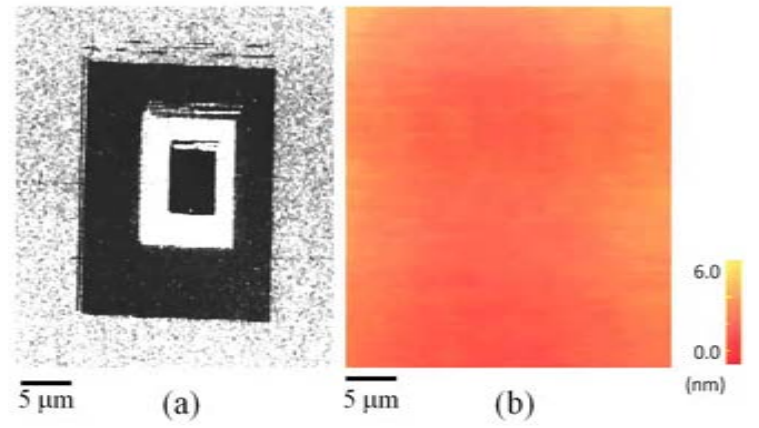

FIG. 11: (a) Conductance image and (b) topographic image of the surface of the PANI film after the electrical modification.
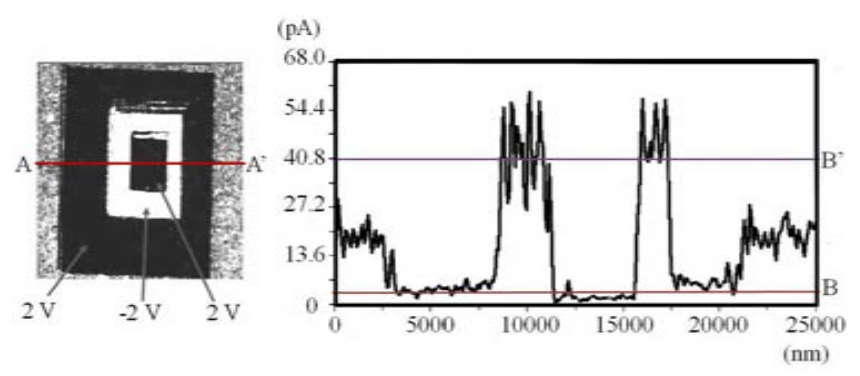

FIG. 12: Current profile of the conductance image at line A-A after the electrical modification. B and B' lines correspond to current averages of the $2 \mathrm{~V}$ and $-2 \mathrm{~V}$ modified area, respectively.

highest value of the conductivity of the PANI. One of the reasons is that this calculated conductivity includes the contact resistance between the tip and the surface of the film. The other reason is that the PANI film was not perfectly changed to conductive state (half oxidized salt state) by the negative voltage of $-2 \mathrm{~V}$. It is expected that the ratio between a high and low conductance area can be increased by optimization of the condition by electrical modification. Therefore, it is expected from these results that this conductive polymer provides rewritable recording material based on the conductance change for scanning multiprobe high-density data storage.

\section{CONCLUSION}

This paper reports the formation of the flat conductive polymer film as a recording media using the surfacegraft polymerization on the template-stripped gold (TSG) for scanning multiprobe data storage. The TSG surface was formed by following process: First, the gold film was deposited on the cleaved mica surface by sputtering. Then, this gold film was transferred from the cleaved mica onto the gold-coated Si substrate using the gold-gold direct bonding technique. It is shown from the AFM and STM measurements that the surface roughness of TSG was much smaller than that of the sputtered gold film. Then, the PANI film as a recording medium was formed on the TSG surface using the surface-graft polymerization. It is demonstrated that the use of the TSG surface is efficient for the formation of the flat conductive polymer film. Then, the reversible conductance switching of the flat PANI film was demonstrated by switching polarity of applying voltage to the film with the groundedconductive tip. This result indicates that conductive polymer has possibility of realizing rewritable recording media for scanning probe data storage system. Conductive polymer recording media with smaller roughness than that of this study may be realized by optimizing the molecular design, the synthesis method and dopant of the conductive polymer. It is believed that the conductive polymer recording media with ultraflat surface can bring highspeed, high-reliability and high-density data storage to scanning multiprobe data storage.

\section{Acknowledgments}

Part of this work was performed in the Micro/Nanomachining Research Education Center (MNC) of Tohoku University. This work was supported in part by a Grant-in Aid for Scientific Research from the Japanese Ministry of Education, Culture, Sports, Science and Technology of Japan, also supported in part by Special Coordination Funds for Promoting Science and Technology, Formation of Innovation Center for Fusion of Advanced Technologies.
[1] J. P. Yang, J. Nanosci. Nanotechnol. 7, 181 (2007).

[2] S. Naberhuis, J. Magn. Magn. Mater. 249, 447 (2002).

[3] H. Shin, K.-M. Lee, W.-K. Moon, J. U. Jeon, G. Lim, Y. E. Pak, J. H. Park, and K. H. Yoon, IEEE Trans. Ultrason. Ferroelectr. Freq. Control. 47, 801 (2000).

[4] Y. L. Shang, Y. Q. Wen, S. L. Li, S. X. Du, X. B. He, L. Cai, Y. F. Li, L. M. Yang, H.J. Gao, and Y. Song, J. Am. Chem. Soc. 129, 11674 (2007).

[5] R. Bennewits, J. N. Crain, A. Kirakosian, J.-L. Lin, J. L. McChesney, D. Y. Petrovykh, and F. J. Himpsel, Nanotechnology 13, 499 (2002).

[6] B. Gotsmann, U. Duerig, J. Frommer, and C. J. Hawker, Adv. Funct. Mater. 16, 1499 (2006).

[7] C. D. Wright, M. Armand, and M. M. Aziz, IEEE Trans. Nanotech. 5, 50 (2006).

[8] R. Pandian, B. J. Kooi, G. Palasantzas, J. T. M. D. Hos- son, and A. Pauza, Adv. Mater. 19, 4431(2007).

[9] K. Tanaka, Y. Kurihashi, T. Uda, Y. Daimon, N. Odagawa, R. Hirose, Y. Hiranaga, and Y. Cho, Jpn. J. Appl. Phys. 47, 3311 (2008).

[10] H. F. Hamann, M. O'Boyle, Y. C. Martin, M. Rooks, K. Wickramasinghe, Nat. Mater. 5, 383 (2006).

[11] S. Hosaka, T. Shintani, M. Miyamoto, A. Hirotsune, M. Terao, M. Yoshida, S. Honma, and S. Kammer, Thin Solid Films 273, 122 (1996).

[12] E.-J. Hong, W.-S. Oh, N.-C. Park, H.-S. Yang, Y.-P. Park, S.-Q. Lee, E.-K. Kim, and K.-H. Park, Jpn. J. Appl. Phys. 44, 3364 (2005).

[13] H. Takahashi, T. Ono, A. Onoe, Y. Cho, and M. Esashi, J. Micromech. Microeng. 16, 1620 (2006).

[14] E. Eleftheriou, T. Antonakopoulos, G. K. Binnig, G. Cherubini, M. Despont, A. Dholakia, U. Durig, M. A. 
Lantz, H. Pozidis, H. E. Rothuizen, and P. Vettiger, IEEE Trans. Magn. 39, 938 (2003).

[15] D.-W. Lee, T. Ono, T. Abe, and M. Esashi, J. Microelectromech. Syst. 11, 215 (2002).

[16] Y.-S. Kim, S. Jang, C. S. Lee, W.-H. Jin, I.-J. Cho, M.-H. Ha, H.-J. Nam, J.-U. Bu, S.-I. Chang, and E. Yoon, Sens. Actuators A: 135, 67 (2007).

[17] S. Yoshida, T. Ono, and M. Esashi, Nanotechnology 18, $505302(2007)$

[18] S. Yoshida, T. Ono, S. Oi, and M. Esashi, Nanotechnology 16, 2516 (2005).

[19] T. Ono, S. Yoshida, and M. Esashi, Nanotechnology 14, 1051 (2003).

[20] M. Hegner, P. Wagner, and G. Semenza, Surf. Sci. 291, 39 (1993).

[21] D. W. Mosley, B. Y. Chow, and J. M. Jacobson, Langmuir 22, 2437 (2006)

[22] P. Wagner, M. Hegner, H.-J. Guntherodt, and G. Semenza, Langmuir 11, 3867 (1995).
[23] S. Lee, S.-S. Bae, G. Medeiros-Ribeiro, J. J. Blackstock, S. Kim, D. R. Stewart, and R. Ragan, Langmuir 24, 5984 (2008).

[24] E. A. Weiss, G. K. Kaufman, J. K. Kriebel, Z. Li, R. Schalek, and G. M. Whitesides, Langmuir 23, 9686 (2007).

[25] R. Ragan, D. Ohlberg, J. J. Blackstock, S. Kim, and R. S. Williams, J. Phys. Chem. B. 108, 20187 (2004).

[26] D. Ohlberg, J. J. Blackstock, R. Ragan, S. Kim, R. S. Williams, Appl. Phys. A 80, 1327 (2005).

[27] E. M. Geniès, A. Boyle, M. Lapkowski, and C. Tsintavis, Synth. Met. 36, 139 (1990)

[28] D. Nicolas-Debarnot and F. Poncin-Epaillard, Anal. Chim. Acta 475, 1 (2003).

[29] M. S. Faizul, T. Ono, Y. Kawai, and M. Esashi, Proceeding of the 11th international Conference on New Actuators, 75 (2008). 